\title{
Mid- and long-term results in patients with thoracic endovascular aortic repair
}

\author{
Yaşar Gökkurt (D), İsmail Yürekli (D), Habib Çakır (D, Hasan İner (D), Nihan Yeşilkaya (D), Ertürk Karaağaç (D), Orhan Gökalp (D), \\ Levent Yllık (D), Ali Gürbüz (D)
}

Department of Cardiovascular Surgery, Izmir Katip Çelebi University, Izmir, Turkey

Received: July 01, 2021 Accepted: July 19, 2021 Published online: August 11, 2021

\begin{abstract}
Objectives: In this study, we aimed to examine the mid- and long-term results of the patients undergoing thoracic endovascular aortic repair (TEVAR) in our clinic.

Patients and methods: Between June 2006 and October 2018, a total of 71 patients (59 males, 12 females; mean age: $60.9 \pm 13.3$ years; range, 17 to 79 years) who underwent TEVAR electively or urgently were retrospectively analyzed. Data including demographic and clinical characteristics of the patients and operative data were recorded.

Results: Of the patients, 22 (31\%) were urgently operated and 49 (69\%) were electively operated. Cerebrovascular disease (CVD) developed in three (4.2\%) patients. Thirty-day overall survival of patients who were operated on emergency was found to be $72.7 \%$, and this rate was $98 \%$ in elective patients ( $\mathrm{p}=0.001$ ). One-year overall survival of our patient series was $76.1 \%$. One-year survival rate of emergency patients was $54.5 \%$, and this rate was $85.7 \%$ in elective patients $(p=0.002)$. Two-year overall survival of emergency patients was $45.5 \%$ and it was $79.6 \%$ in elective patients $(\mathrm{p}=0.002)$. While the survival rate of preoperative smokers after two years was $60.8 \%$, it was calculated as $90 \%$ of preoperatively non-smokers $(\mathrm{p}=0.022)$.

Conclusion: Thoracic endovascular aortic repair treatment seems to show similar results to open surgery in terms of mortality and other complications in the mid- and long-term. With the developing stent-graft technology, TEVAR results can be even better in the future.
\end{abstract}

Keywords: Mortality, thoracic aortic aneurysm, thoracic endovascular aortic repair.

Standard conventional surgical treatment of thoracic aortic aneurysms (TAAs) is still associated with high mortality and morbidity. The risks of open surgical methods are quite high due to factors such as long clamping times, cardiac stress during clamping, protection of the medulla spinalis, and the use of extra-corporeal circulation. Alternative methods have been sought in the treatment of aortic aneurysms to minimize these risks. For the first time in 1991, Parodi et al. ${ }^{[1]}$ reported successful endovascular treatment in patients with abdominal aortic aneurysms. Over time, endovascular stent grafts have started to be used in patients with TAA, and very good results have been reported, particularly in the early period. ${ }^{[2,3]}$ One of the most important disadvantages in the applicability of endovascular treatment is that the patient requires anatomical compatibility. The scope of anatomical suitability criteria has expanded due to the recent advances in technology and increasing surgical experience. In addition, thoracic endovascular aortic repair (TEVAR) has been applied to some patients who are anatomically unsuitable with hybrid procedures.
The main disadvantages of TEVAR are endoleaks and graft migration. Currently, endovascular methods are widely used as an alternative to classical surgical treatment thanks to the developing technology, increasing surgical experience, the introduction of hybrid procedures, and the advantages it provides in the early period. However, in the long term, TEVAR seems to lose its advantages due to endoleak in the early and mid-term due to problems such as graft migration and the growth of aneurysm sac.

In this study, we aimed to examine the mid- and long-term results of patients undergoing TEVAR in our clinic.

Corresponding author: Yaşar Gökkurt, MD. İzmir Katip Çelebi Üniversitesi Tıp Fakültesi Kalp ve Damar Cerrahisi Anabilim Dalı, 35620 Çiğli, İzmir, Türkiye. Tel: +90 506 - 7619953 e-mail: yasargokkurt@hotmail.com

\section{Citation:}

Gökkurt Y, Yürekli İ, Çakır H, İner H, Yeşilkaya N, Karaağaç E, et al. Mid- and long-term results in patients with thoracic endovascular aortic repair. Cardiovasc Surg Int 2021;8(2):103-108. 


\section{PATIENTS AND METHODS}

This single-center, retrospective study was conducted at Izmir Atatürk Training and Research Hospital, Cardiovascular Surgery Clinic between June 2006 and October 2018. A total of 83 patients who underwent TEVAR electively or urgently were screened. Of these patients, 12 were excluded from the study due to missing data. No other exclusion criteria were applied. Finally, a total of 71 patients (59 males, 12 females; mean age: $60.9 \pm 13.3$ years; range, 17 to 79 years) were included in the study. A written informed consent was obtained from each patient. The study protocol was approved by the Izmir Katip Celebi University, Faculty of Medicine, Clinical Research Ethics Committee (date, no 18.06.2020, 0228). The study was conducted in accordance with the principles of the Declaration of Helsinki.

Data including age, sex, smoking history, hypertension, diabetes mellitus (DM), chronic obstructive pulmonary disease (COPD), previous cerebrovascular disease (CVD) and prior cardiovascular disease were recorded. On preoperative computed tomography (CT), the aneurysm and normal aortic diameters, pre- and postoperative laboratory blood values, transthoracic echocardiography findings, proximal and distal landing zone and diameters, oversize percentages, extubation, intensive care unit (ICU) stay and discharge times, endoleak development, and type and use of additional stent graft, the need for additional surgical interventions, need for debranching, blood product requirement and amount, stent graft diameters, and mortality rates were evaluated. All data were collected using the file archive of our clinic and the computer records of the patients. The patients who were asymptomatic and had degenerative or traumatic aneurysms exceeding $5.5 \mathrm{~cm}$, the patients who had saccular aneurysms, and all symptomatic TAAs were managed by endovascular techniques.

General anesthesia was administered to the majority of the patients. The patients with comorbidities underwent local anesthesia + sedation. Following proper site cleaning, all patients were covered using sterile drapes. In the surgical method we used, the access route was preferred as the femoral region in all our patients. Common femoral artery, superficial femoral artery, and profunda femoris artery were rotated one by one with nylon tape through a femoral incision from the side with suitable iliac anatomy. After the femoral arteriotomy, the hydrophilic guidewire was advanced over the catheter from the ascending to the aorta. In most cases, the Medtronic ${ }^{\circledR}$ (Medtronic Inc., Minneapolis, MN, USA) graft brand was used, as it was easier to reach these grafts in emergency cases. In 18 cases, the left subclavian artery was closed in our endovascular graft application and three cases required left subclavian bypass. No fenestrated graft was used in any of the cases.

\section{Statistical analysis}

Statistical analysis was performed using the IBM SPSS for Windows version 22.0 software (IBM Corp., Armonk, NY, USA). Descriptive data were expressed in mean \pm standard deviation (SD) for normally distributed variables and in median (min-max) for non-normally distributed variables. Categorical variables were expressed in number and frequency. The Pearson chi-square and Fisher's exact tests were used to analyze categorical variables. The compliance of continuous variables to normal distribution was examined using visual (histogram) and analytical methods (Kolmogorov-Smirnov/Shapiro-Wilk tests). The t-test was used for the comparison of normally distributed variables between two groups, and the Mann-Whitney $U$ test was used for the comparison of variables that did not show normal distribution between the two groups. The survival rates were calculated using the Kaplan-Meier survival analysis. The effects of variables on survival were analyzed using the log-rank test. Possible factors whose effects on survival were analyzed in the univariate analysis were included in the Cox regression analysis with backward and enter selection methods. A $p$ value of $<0.05$ was considered statistically significant.

\section{RESULTS}

Baseline demographic and clinical characteristics of the patients are shown in Table 1 . Of the patients, $22(31 \%)$ were urgently operated and $49(69 \%)$ were electively operated. Of the patients, four (5.6\%) had a saccular aneurysm and 67 (94.4\%) had a fusiform aneurysm.

The median follow-up was 51.3 (range, 17 to 78 years) months. Of the patients included in the study, $53(74.6 \%)$ were smoking and $25(35.2 \%)$ had a history of COPD. Nineteen (26.8\%) of the patients had DM, 25 (35.2\%) had hyperlipidemia, 15 (21.1\%) had a history of chronic renal failure, 14 (19.7\%) had coronary artery disease, 
$16(22.5 \%)$ had peripheral artery disease, and six (8.5\%) had a history of previous CVD (Table 1). Blood product was used in seven patients. A total of 28 units of blood products were used. The median length of stay in the ICU was 3.4 (range, 1 to 28) days. While general anesthesia was applied to 64 of the patients, TEVAR operation was performed under local anesthesia and sedation for seven of them. Proximal landing zone was 0 in one patient (1.4\%), 1 in four patients (5.6\%), 2 in 13 patients (18.3\%), 3 in 36 patients $(50.7 \%), 4$ in 17 patients (24\%) according to the Ishimaru classification. The mean proximal landing zone diameter was $31.1 \pm 5.5 \mathrm{~mm}$, while the mean distal landing zone diameter was $30.8 \pm 7.3 \mathrm{~mm}$.

No additional surgical intervention was performed in $63(88.7 \%)$ of the patients. Carotico-subclavian bypass was performed in five (7\%) patients, and subclavian-subclavian bypass was performed in three (4.2\%) patients. Celiac artery was closed in only two $(2.8 \%)$ of the cases (Table 2).

\begin{tabular}{|c|c|c|}
\hline \multicolumn{3}{|c|}{ Table 1} \\
\hline Variables & $\mathrm{n}$ & $\%$ \\
\hline \multicolumn{3}{|l|}{ Age (year) } \\
\hline $17-40$ & 6 & 8.4 \\
\hline $41-75$ & 61 & 86 \\
\hline$>75$ & 4 & 5.6 \\
\hline \multicolumn{3}{|l|}{ Sex } \\
\hline Male & 59 & 83.1 \\
\hline Smoking history & 53 & 74.6 \\
\hline \multicolumn{3}{|l|}{ Associated diseases } \\
\hline Hypertension & 58 & 81.7 \\
\hline COPD & 25 & 35.2 \\
\hline Diabetes mellitus & 19 & 26.8 \\
\hline Hyperlipidemia & 25 & 35.2 \\
\hline Chronic renal failure & 15 & 21.1 \\
\hline Coronary artery disease & 14 & 19.7 \\
\hline Peripheral artery disease & 16 & 22.5 \\
\hline Previous CVD & 6 & 8.5 \\
\hline Previous cardiac operations & 13 & 18.3 \\
\hline \multicolumn{3}{|l|}{ Etiology in cases } \\
\hline Degenerative aneurysm & 53 & 74.6 \\
\hline Dissecting aneurysm & 7 & 9.9 \\
\hline Dissection & 1 & 1.4 \\
\hline Trauma & 5 & 7 \\
\hline Connective tissue disease & 5 & 7 \\
\hline
\end{tabular}

Additional balloons were required in 32 (45.1\%) of the patients. While no endoleak developed in $52(73.2 \%)$ patients on angiography performed after the procedure, 19 patients (26.8\%) developed endoleaks.

\begin{tabular}{|c|c|c|}
\hline \multicolumn{3}{|c|}{$\begin{array}{c}\text { Table } 2 \\
\text { Operative data }(n=71)\end{array}$} \\
\hline Variables & $\mathrm{n}$ & $\%$ \\
\hline Emergency & 22 & 31 \\
\hline Elective & 49 & 69 \\
\hline $\begin{array}{l}\text { Anesthesia } \\
\text { General } \\
\text { Local + sedation }\end{array}$ & $\begin{array}{c}64 \\
7\end{array}$ & $\begin{array}{c}90.1 \\
9.9\end{array}$ \\
\hline $\begin{array}{l}\text { Graft brand } \\
\text { Medtronic } \\
\text { Jotec } \\
\text { GORE } \\
\text { Cook } \\
\text { Relay }\end{array}$ & $\begin{array}{c}49 \\
2 \\
16 \\
1 \\
3\end{array}$ & $\begin{array}{c}69 \\
2.8 \\
22.5 \\
1.4 \\
4.2\end{array}$ \\
\hline Proximal lengthening & 3 & 4.2 \\
\hline Distal lengthening & 8 & 11.3 \\
\hline Additional balloon & 32 & 45.1 \\
\hline Debranching & 5 & 7 \\
\hline Frozen elephant trunk & 4 & 5.6 \\
\hline LSA closure & 18 & 25.4 \\
\hline Celiac artery closure & 2 & 2.8 \\
\hline Proximal landing zone & & \\
\hline 0 & 1 & 1.4 \\
\hline 1 & 4 & 5.6 \\
\hline 2 & 13 & 18.3 \\
\hline 3 & 36 & 50.7 \\
\hline 4 & 17 & 24 \\
\hline
\end{tabular}

\begin{tabular}{|lcc|}
\multicolumn{1}{c}{ Table 3 } & & \\
\multicolumn{1}{|c|}{ Postoperative data $(\mathrm{n}=71)$} & & \\
\hline Variables & $\mathrm{n}$ & $\%$ \\
\hline Postoperative complications & & \\
$\quad$ Acute renal failure & 7 & 9.9 \\
Wound infection & 9 & 12.7 \\
Cerebrovascular diseases & 7 & 9.9 \\
Pneumonia & 9 & 12.7 \\
Additional surgical intervention & & \\
Carotico-subclavian bypass & 5 & 7 \\
Subclavian-subclavian bypass & 3 & 4.2 \\
Open surgery & 1 & 1.4 \\
Endoleak type & & \\
1 & 16 & 22.5 \\
2 & 3 & 4.2 \\
\hline
\end{tabular}




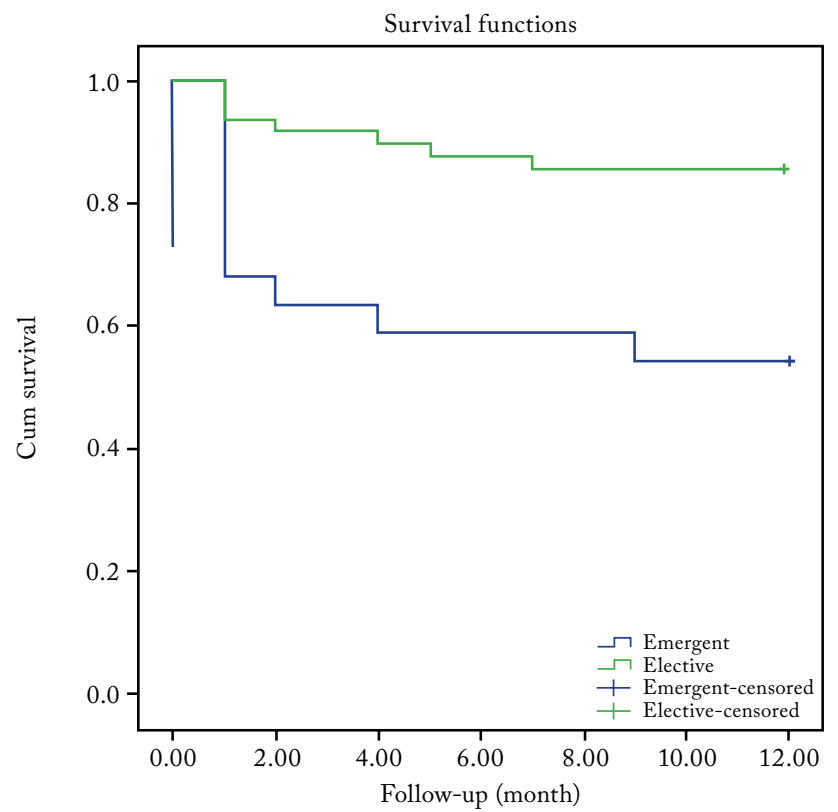

Figure 1. Kaplan-Meier survival analysis comparing elective and emergent cases in one year.

Type 1 endoleak developed in 16 patients and type 2 endoleak in three patients.

There were seven (9.9\%) patients who developed acute renal failure, nine (12.7\%) patients developed wound infection, seven (9.9\%) patients developed CVD, and nine (12.7\%) patients developed pneumonia at the postoperative first month (Table 3 ). In the postoperative follow-up, a total of three patients had permanent paresis and/or plegia.

The 30-day survival of the patients who were operated urgently was $72.7 \%$, and this rate was $98 \%$ in elective patients $(\mathrm{p}=0.001)$. The one-year survival of our patient series was $76.1 \%$. The two-year survival was $69.5 \%$ and the overall survival was $42.4 \%$. The one-year survival rate of emergency patients was $54.5 \%$, and this rate was $85.7 \%$ for elective patients $(\mathrm{p}=0.002)$. Emergency operation increases the one-year mortality risk by 5.6 times (Figure 1), COPD by 6.4 times, hyperlipidemia by 3.3 times. The two-year survival of emergency patients was $45.5 \%$, and this rate was $79.6 \%$ in elective patients $(\mathrm{p}=0.002)$ (Figure 2). The two-year survival of smokers was $60.8 \%$, while it was $90 \%$ for non-smokers $(\mathrm{p}=0.022)$. The two-year survival of patients with and without COPD was $40 \%$ and $84.8 \%$, respectively $(\mathrm{p}<0.001)$. According

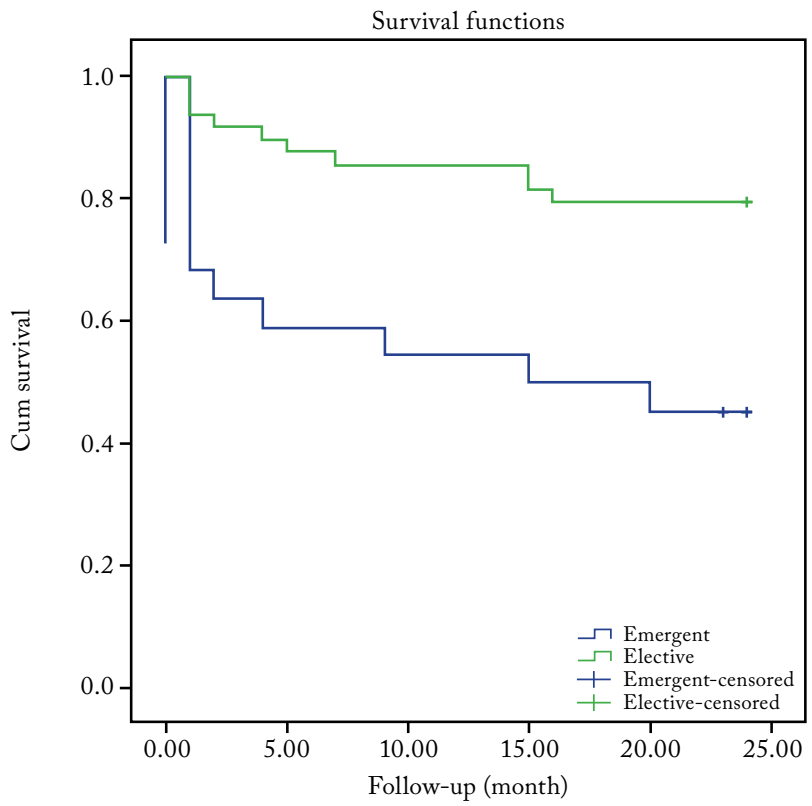

Figure 2. Kaplan-Meier survival analysis comparing elective and emergent cases in two years.

to the analysis, emergency operation increased the two-year mortality risk by 3.9 times compared to elective operations $(p=0.002)$, and COPD increased by 6.2 times in the same way. Each unit increase in age at five-year survival increased the risk of mortality by $4 \%$ (odds ratio [OR]: 1.04 ). While the five-year survival rate of male patients was found to be $33.9 \%$, this rate was $83.3 \%$ in females $(\mathrm{p}=0.008)$. Five-year survival rate of smokers and non-smokers was $28.6 \%$ and $78.7 \%$, respectively $(\mathrm{p}=0.001)$. The five-year survival of patients with and without DM was $14 \%$ and $51.8 \%$, respectively $(\mathrm{p}<0.001)$. The five-year survival of patients with and without hyperlipidemia was $25.7 \%$ and $51.6 \%(\mathrm{p}=0.017)$. The five-year survival of the patients with and without COPD was $8.9 \%$ and $61.3 \%$, respectively $(\mathrm{p}<0.001)$.

\section{DISCUSSION}

The primary objective of this study is to investigate the effect of TEVAR on mortality, particularly in the mid- and long-term. In our study, one-year survival was $76.1 \%$, two-year survival was $69 \%$, and five-year survival was $42.4 \%$. In our study, mortality in the emergency TEVAR procedure was 17.1 times higher than in the elective TEVAR. 
In a systematic review and meta-analysis on TEVAR treatment in degenerative aneurysms, Biancari et al. ${ }^{[4]}$ found 30 -day mortality to be $4 \%$ and one-year mortality to be $19.7 \%$. In a meta-analysis, 30 -day mortality was found to be $19 \%$ in patients who underwent TEVAR with a ruptured TAA. ${ }^{[5]}$ In our study, the 30-day mortality was $2 \%(n=1)$ in patients who underwent elective TEVAR, indicating a lower rate than reported in the literature. This patient, who had exitus, died from acute renal failure on the postoperative fifth day. In a multi-center study, 30-day mortality was found to be $27.9 \%$ in patients with a ruptured TAA who underwent emergency TEVAR. ${ }^{[6]}$ In our study, the 30 -day mortality was $27.3 \%(n=6 / 22)$ in patients who underwent emergency TEVAR, consistent with the literature data. The median time to death for our patients who were taken to emergency TEVAR and died was six (range, 0 to 10 ) days. We believe that, in the cox regression analysis performed with five parameters affecting 30-day mortality, emergency TEVAR application increased the mortality by 17.1 times compared to elective TEVAR procedure. In the aforementioned meta-analysis, the first 30-day mortality in emergency patients who underwent open surgery reached up to $33 \% .{ }^{[5]}$ In our study, although there were patients with severe hypotensive shock in the emergency TEVAR group, the mortality rate was lower than the first 30-day open surgery mortality rate reported in this meta-analysis. In our study, one-year survival rate was $76.1 \%$. In the cox regression analysis of one-year survival; the emergency TEVAR procedure increased the one-year mortality by 5.6 times. In addition, unlike 30-day mortality, COPD increased one-year mortality by 6.4 times and hyperlipidemia by 3.3 times. In our study, two-year survival rate was $69 \%$ (45.5\% of emergency patients and $79.6 \%$ of elective patients). When the factors affecting two-year survival were analyzed, COPD increased two-year mortality by 6.2 times and emergency TEVAR by 3.9 times.

In a study conducted by Schaffer et al., ${ }^{[7]} 11,996$ patients who underwent TEVAR for various reasons were evaluated. The five-year survival rate was calculated as $60 \%$ in patients treated with TEVAR. In our study, the five-year survival rate was $42.4 \%$. When one-year and five-year causes of death were examined in our study, none of the causes of death were the aneurysms or events related to the TEVAR procedure. While the most common cause of death at one year was myocardial infarction, malignancy was the most common cause of death at five years. In our study, since there was no aneurysm-related death in the mid- and late periods; our five-year survival rate is lower than the literature, suggesting that the causes of comorbidities may be higher in patients included in the study. When the factors affecting five-year mortality were examined in our study, the most important factors were advanced age and male sex. Other factors were DM (increased mortality by 2.8 times) and smoking (increased mortality by 3.1 times).

Another focus of the discussions in the literature is whether sex has an effect on mortality. Deery et al. ${ }^{[8]}$ reported $12 \%$ mortality in female patients and $8 \%$ in male patients during one-year follow-up of patients who underwent TEVAR. They found a statistically significantly higher mortality rates among female patients. In our study, there was no significant difference in terms of 30-day mortality, and one- and two-year mortality between male and female patients. However, when the five-year mortality was examined using the Cox regression analysis, male sex increased the five-year mortality by 4.3 times compared to female sex. Considering the causes of death of our patients, the atherosclerotic process stands out which may explain the higher frequency of mortality in men, particularly in the long-term.

There are different findings in the literature when other factors affecting mortality in patients treated with TEVAR are examined. In a study conducted by Geisbüsch et al., ${ }^{[9]}$ risk factors for mortality after TEVAR were reported as renal failure, over 75 years of age, and emergency operations as independent risk factors. Chung et al. ${ }^{[10]}$ defined the presence of preoperative leukocytosis and aneurysm diameter as independent risk factors for late mortality. Wang et al. ${ }^{[11]}$ reported the results of TEVAR in patients with impaired renal function and that emergency patients with a creatinine value of $>2 \mathrm{mg} / \mathrm{dL}$ had a poor prognosis. Dillavou and Makaroun ${ }^{[12]}$ also reported that increased preoperative creatinine levels had a predictive value for mortality or morbidity endpoints. Salihi et al. ${ }^{[13]}$ showed that early mortality developed due to low cardiac output syndrome. In a meta-analysis by Harky et al., ${ }^{[14]}$ when the patients who underwent TEVAR and those who underwent open surgery were compared, the one-year mortality rate was $22.19 \%$ and the five-year mortality rate was $44.26 \%$ in 3,908 patients who underwent TEVAR. In 10,672 patients who underwent open surgery, the one-year mortality rate was $24.04 \%$, and the five-year mortality rate was $37.37 \%$. When these results were compared, no 
significant difference was found between one-year and five-year mortality rates. In our study, one-year mortality in patients who underwent TEVAR was found to be $23.9 \%$. The five-year survival rate was found to be $42.4 \%$. Considering five-year mortality rates in our study versus the literature data, the deaths at five years were not associated with an aneurysm. We believe that TEVAR would not lose its advantage in the mid- and long-term in the near future. The secondary objective of our study was to evaluate midand long-term complications of the TEVAR procedure. In particular, the patients who required paresis, plegia, endoleaks, and aneurysm-related reintervention after discharge. In the meta-analysis by Biancari et al., ${ }^{[4]}$ the rate of postoperative paraplegia was found to be $3.2 \%$ and the rate of permanent paraplegia was found to be $1.4 \%$ in patients who received TEVAR due to TAA. The formation rate of CVD was calculated $2.7 \%$. In our study, plegia/paresis occurred in seven patients in the postoperative period (9.9\%). In one of these patients, plegia was permanent (1.40\%). Three patients developed CVD, and the rate of CVD occurrence was calculated as $4.2 \%$.

There are several limitations to this study. First, since the tomography data of some postoperative patients could not be accessed, these patients were excluded from the study. Second, the postoperative opaque nephropathy relationship could not be established, as the data on how much opaque material were used during the operation could not be reached in most patients. Since it is a retrospective study, we could not reach some patients such as tomography, blood results, and postoperative follow-up and, therefore, we had to reduce the number of patients in the study.

In conclusion, TEVAR treatment seems to show similar results to open surgery in terms of mortality and other complications in the mid- and long-term. It seems that TEVAR treatment does not lose its obvious advantage in the early period and, also, in the mid- and long-term. With the developing stent-graft technology, TEVAR results can be even better in the future.

\section{Declaration of conflicting interests}

The authors declared no conflicts of interest with respect to the authorship and/or publication of this article.

\section{Funding}

The authors received no financial support for the research and/or authorship of this article.

\section{REFERENCES}

1. Parodi JC, Palmaz JC, Barone HD. Transfemoral intraluminal graft implantation for abdominal aortic aneurysms. Ann Vasc Surg 1991;5:491-9.

2. Fairman RM, Criado F, Farber M, Kwolek C, Mehta M, White $\mathrm{R}$, et al. Pivotal results of the medtronic vascular talent thoracic stent graft system: The VALOR trial. J Vasc Surg 2008;48:546-54.

3. Nauta FJ, Trimarchi S, Kamman AV, Moll FL, van Herwaarden JA, Patel HJ, et al. Update in the management of type B aortic dissection. Vasc Med 2016;21:251-63.

4. Biancari F, Mariscalco G, Mariani S, Saari P, Satta J, Juvonen T. Endovascular treatment of degenerative aneurysms involving only the descending thoracic aorta: Systematic review and meta-analysis. J Endovasc Ther 2016;23:387-92.

5. Naughton PA, Park MS, Morasch MD, Rodriguez HE, Garcia-Toca M, Wang CE, et al. Emergent repair of acute thoracic aortic catastrophes. A Comparative Analysis. Arch Surg 2012;147:243-9.

6. López Espada C, Linares Palomino JP, Domínguez González JM, Iborra Ortega E, Lozano Vilardell P, Solanich Valldaura $\mathrm{T}$, et al. A multicenter study of emergency endovascular repair of the thoracic aorta: Indications and outcomes. Med Intensiva (Engl Ed) 2021;45:280-8.

7. Schaffer JM, Lingala B, Miller DC, Woo YJ, Mitchell RS, Dake MD. Midterm survival after thoracic endovascular aortic repair in more than 10,000 Medicare patients. J Thorac Cardiovasc Surg 2015;149:808-20.

8. Deery SE, Shean KE, Wang GJ, Black JH 3rd, Upchurch GR Jr, Giles KA, et al. Female sex independently predicts mortality after thoracic endovascular aortic repair for intact descending thoracic aortic aneurysms. J Vasc Surg 2017;66:2-8.

9. Geisbüsch P, Kotelis D, Hyhlik-Dürr A, Hakimi M, Attigah N, Böckler D. Endografting in the aortic arch - does the proximal landing zone influence outcome? Eur J Vasc Endovasc Surg 2010;39:693-9.

10. Chung J, Corriere MA, Veeraswamy RK, Kasirajan K, Milner R, Dodson TF, et al. Risk factors for late mortality after endovascular repair of the thoracic aorta. J Vasc Surg 2010;52:549-54.

11. Wang GJ, Fairman RM, Jackson BM, Szeto WY, Pochettino A, Woo EY. The outcome of thoracic endovascular aortic repair (TEVAR) in patients with renal insufficiency. J Vasc Surg 2009;49:42-6.

12. Dillavou ED, Makaroun MS. Predictors of morbidity and mortality with endovascular and open thoracic aneurysm repair. J Vasc Surg 2008;48:1114-9.

13. Salihi S, Saçlı H, Erkengel Hİ, Kara İ. Mid-term outcomes of thoracic endovascular aortic repair for complicated type B aortic dissection. Turk J Vasc Surg 2020;29:26-31.

14. Harky A, Kai Chan JS, Ming Wong CH, Bashir M. Open versus endovascular repair of descending thoracic aortic aneurysm disease: A systematic review and meta-analysis. Ann Vasc Surg 2019;54:304-15. 